vigor has yielded beneath the influence of want or chronic disease, in the aged, or the young grown prematurely old by over-application or constitutional decay, and in the dissipated, whose pliysical constitution is as degraded as their moral attributes. In all such cases, the paralyzing influence of this agent on a fatty, feeble heart may be feared, and its use should be avoided. With the precautions we have insisted on, the administration of chloroform will prove a blessing; without them, its abuse threatens to be a curse.-London Lancet.

\title{
A CASE OF QUADRUPLE BIRTH.
}

BY S. KENNERLY, M.D., AUgUSTA COUNTY, VIRGINIA.

$\mathrm{ON}_{\mathrm{N}}$ the night of the 5th of August, I was called to see Mrs. W., supposed to be in labor. On my arrival I was informed by my friend, Dr. A. Waddell, who had also been called in, that he had just delivered the head of a child, which had been expelled feet foremost, the head being retained. He was told that it had been in that position for two hours. Upon examination, we found that there was a second child inclosed in its membranes; and as there had been no recurrence of pain since the partial expulsion of the first child, and the mouth of the womb being entirely relaxed, we concluded that the safest plan would be to administer ergot, and complete the delivery. We accordingly gave her $3 \mathrm{j}$. of liquor ergotina, prepared by Purcell, Ladd \& Co., which quickened and increased her pain; and in three quarters of an hour the second child was expelled, followed by a subsidence of pain. On examination, we found a third child presenting, breech foremost, with no membranes around it. After waiting half an hour, and no pain recurring, we brought down the feet, and repeated the liquor ergotina in the dose of 3 ss., and in half an hour the third child was expelled. On examination, we found a fourth child presenting, head foremost, which was expelled in about twenty minutes, inclosed in its proper membranes. There were two distinct placentas, and they soon followed the birth of the fourth child. One was very large, and divided into three distinct lobes, each lobe having its respective cord attached to its centre. The smaller placenta was a little larger than either lobe of the larger placenta. The children, three boys and a girl, were all born alive, but neither lived over fifteen minutes. I suppose they were not viable, for they were evidently born too soon, though they were perfect in every respect, so far as $I$ could judge. The finger and toe-nails were perfect. They weighed nearly eight pounds next morning, and measured sixteen inches in length. Mrs. W. did not expect to be confined until the latter part of October next, and thinks she quickened about the middle of June. So the children must have been under seven months. She did not suffer as much as in either of two former labors of single births, and is doing remarkably well. What seems a little singular is, that there should have been a total suspension of uterine contractions for two hours after expulsion of the first child, even while its head remained in the vulva, and the second child protruding from the womb into the vagina. 
I think that the second and third children were inclosed in the same membranes, though their cords were each attached to its respective lobe of the larger placenta. There was but little hemorrhage, and the womb contracted well after the expulsion of the placentas.

Since the occurrence of the above case, I have attended three other women in confinement, two of whom had twins ; neither of which cases possessed any particular interest.-The Stethoscope.

\section{OBSTRUCTION AND PERFORATION OF THE BOWELS.}

[Communicated for the Boston Medical and Surgical Journal.]

As "when sorrows come, they come not single spies, but in battalions ;" so when the young physician's ill luck comes, it comes not singly, but in groups. Very recently, it was my fortune to report the fatal termination of a case of parturition, complicated with a sanguineous tumor of the labia. I have now to report another fatal case, to me quite as novel and interesting.

Thursday evening, Nov. 9, I was called to see Joseph Legg, æt. 10, of slender constitution. He had been sick three days, but had had no medical treatment. Upon examination, I found a hard tumor, three by four inches in extent, in the right iliac region, near the ileo-cœcal union. This was quite tender to the touch. No tenderness of the bowels except in this region. Bowels regular, tongue slightly coated, pulse 110. On close examination I could detect no difficulty except at the point above referred to. Gave sub. mur. hyd., grs. v. ; ol. ric., 3 ss.

Friday, Nov. 10th, I found the cathartic had acted, producing a copious watery evacuation, with but little fecal matter. The tumor still existed and was somewhat painful. Ol. ricini was again given, and pulv. Dov. to relieve the pain.

Saturday, 11 th. -1 found the oil had produced free fæcal evacuations, but no diminution in the size of the tumor. Laxatives and anodynes were ordered.

Sunday my patient was more comfortable. Bowels open, tongue cleaning, pulse less than 100, and the tumor less tender and painful. Laxatives and anodynes were continued in diminished doses.

Sunday evening I was called in haste to see the boy. Found him greatly prostrated ; extremities cold ; cold perspiration on face ; respiration hurried; pulse 120, and almost imperceptible ; delirious ; constantly spitting up a frothy substance, with clots of blood; deglutition very difficult; abdomen somewhat swollen and tender, especially over the tumor, and everything indicating a speedy dissolution. Was told that he had, an hour previously, vomited a large amount-a pint at least-of black bloody matter, of a fæcal odor and appearance. Perforation was suspected; and as death was hourly looked for, but little save palliatives was given.

To my surprise I found that my patient was living on Monday, and that he had rallied a little from the prostration of the previous evening. Symptoms of general peritoneal inflammation began to appear. Gave 